\title{
Polarization singularities of focused, radially polarized fields
}

\author{
R. W. Schoonover and T. D. Visser \\ Dept. of Physics and Astronomy, Free University, \\ De Boelelaan 1081, 1081 HV Amsterdam, The Netherlands \\ tvisser@nat.vu.nl
}

\begin{abstract}
The state of polarization of strongly focused, radially polarized electromagnetic fields is examined. It is found that several types of polarization singularities exist. Their relationship is investigated, and it is demonstrated that on smoothly varying a system parameter, such as the aperture angle of the lens, different polarization singularities can annihilate each other. For example, the evolution of a lemon into a monstar and its subsequent annihilation with a star is studied. Also, the quite rare collision of a $C$-line and an $L$-line, resulting in a $V$-point, is observed.
\end{abstract}

(C) 2006 Optical Society of America

OCIS codes: (050.1960) Diffraction theory; (140.3300) Laser beam shaping; (260.2110) Electromagnetic theory; (260.5430) Polarization; (999.9999) Singular optics

\section{References and links}

1. J.F. Nye and M.V. Berry, "Dislocations in wave trains", Proc. R. Soc. Lond. A 336, 165-190 (1974).

2. J.F. Nye, Natural Focusing and Fine Structure of Light (IOP Publishing, Bristol, 1999).

3. M.S. Soskin and M.V. Vasnetsov, in Progress in Optics, edited by E. Wolf (Elsevier, Amsterdam, 2001), Vol. 42.

4. G.P. Karman, M.W. Beijersbergen, A. van Duijl, and J.P. Woerdman, "Creation and annihilation of phase singularities in a focal field," Opt. Lett. 22, 1503-1505 (1997).

5. H.F. Schouten, T.D. Visser, D. Lenstra and H. Blok, "Light transmission through a sub-wavelength slit: waveguiding and optical vortices," Phys. Rev. E 67, 036608 (2003).

6. H.F. Schouten, G. Gbur, T.D. Visser, D. Lenstra and H. Blok, "Creation and annihilation of phase singularities near a sub-wavelength slit," Opt. Express 11, 371-380 (2003)

7. H.F. Schouten, T.D. Visser, G. Gbur, D. Lenstra and H. Blok, "Diffraction of light by narrow slits in plates of different materials," J. Opt. A 6, S277-S280 (2004).

8. D.W. Diehl and T.D. Visser, "Phase singularities of the longitudinal field components in high-aperture systems," J. Opt. Soc. Am. A 21, 2103-2108 (2004).

9. H.F. Schouten, G. Gbur, T.D. Visser and E. Wolf, "Phase singularities of the coherence functions in Young's interference pattern,” Opt. Lett. 28, 968-970 (2003).

10. G. Gbur and T.D. Visser, "Coherence vortices in partially coherent beams," Opt. Commun. 222, 117-125 (2003).

11. D.G. Fischer and T.D. Visser, "Spatial correlation properties of partially coherent focused fields," J. Opt. Soc. Am. A 21, 2097-2102 (2004).

12. G. Gbur and T.D. Visser, "Phase singularities and coherence vortices in linear optical systems," Opt. Commun. 259, 428-435 (2005).

13. M. Born and E. Wolf, Principles of Optics: Electromagnetic Theory of Propagation, Interference and Diffraction of Light, seventh (expanded) ed. (Cambridge University Press, Cambridge, 1999).

14. M.V. Berry and M.R. Dennis, "Polarization singularities in isotropic random vector waves," Proc. R. Soc. Lond. A 457, pp. 141-155 (2001).

15. M.R. Dennis, "Polarization singularities in paraxial vector fields: morphology and statistics," Opt. Commun. 213, pp. 201-221 (2002).

16. I. Freund, A.I. Mokhun, M.S. Soskin, O.V. Angelsky, and I.I. Mokhun, "Stokes singularity relations," Opt. Lett. 27, pp. 545-547 (2002).

17. M.S. Soskin, V. Denisenko, and I. Freund, "Optical polarization singularities and elliptic stationary points," Opt. Lett. 28, pp. 1475-1477 (2003).

$\# 70204$ - \$15.00 USD

(C) 2006 OSA
Received 24 April 2006; revised 17 May 2006; accepted 17 May 2006 12 June 2006 / Vol. 14, No. 12 / OPTICS EXPRESS 5733 
18. A.I. Mokhun, M.S. Soskin, and I. Freund, "Elliptic critical points: C -points, a -lines, and the sign rule," Opt. Lett. 27, pp. 995-997 (2002).

19. T.D. Visser and J.T. Foley, "On the wavefront-spacing of focused, radially polarized beams," J. Opt. Soc. Am. A 22, pp. 2527-2431 (2005).

20. D.W. Diehl, R.W. Schoonover and T.D. Visser, "The structure of focused, radially polarized fields," Opt. Express 14, pp. 3030-3038 (2006),

21. B. Richards and E. Wolf, "Electromagnetic diffraction in optical systems II. Structure of the image field in an aplanatic system," Proc. Royal Soc. A 253, pp. 358-379 (1959).

22. J.D. Jackson, Classical Electrodynamics, $2^{\text {nd }}$ ed. (Wiley, New York, 1975), Sec. 7.2.

23. V.G. Denisenko, R.I. Egorov and M.S. Soskin, "Measurement of the morphological forms of polarization singularities and their statistical weight in optical vector fields," JETP Lett. 80, pp. 17-19 (2004).

24. I. Freund, "Polarization singularity indices in Gaussian laser beams," Opt. Commun. 201, pp. 251-270 (2002).

25. E.T. Copson, An Introduction to the Theory of Functions of a Complex Variable (Oxford University Press, London, 1935). See especially Sec. 4.51.

\section{Introduction}

At points in complex-valued scalar fields where the amplitude is zero, the phase of the field is undetermined or singular [1]. Singular optics is concerned with the description and classification of the different kinds of singularities that can occur in wave fields [2, 3]. Examples of such singularities are the zeros of intensity that are found in focused fields [4]. In real-valued, two-dimensional vector fields, the orientation of the vector is singular wherever the vector vanishes. Such singularities of the Poynting vector field in two-dimensional geometries are studied in Refs. [5]-[7]. Complex-valued vector fields can display singularities of the vector components. An example of these are singularities of the longitudinal component of the electric field in strongly focused, linearly polarized beams [8]. Recently, the two-point correlation functions that describe spatially partially coherent light were shown to posses singularities as well [9][12]. All types of singularities mentioned above can be created or annihilated when a system parameter, such as the wavelength of the field, is smoothly varied.

At every point in a time-harmonic electromagnetic field, the end point of the electric field vector traces out an ellipse as time progresses [13, Sec. 1.4]. The polarization is said to be singular at points where this ellipse degenerates into a circle (at so-called $C$-points) or into a line (at so-called $L$-lines). Polarization singularities in wave fields are described in Refs. [2], and [14]-[18].

Because of their use in, for example, optical trapping, the properties of focused, radially polarized beams have been studied extensively in the past few years (see, e.g., [19] and the references therein). The electric field in the focal region of such a beam has two non-zero parts, namely a radial component and a longitudinal component. The creation and annihilation of phase singularities of these field components has been described in Ref. [20]. It the present paper the rich polarization behavior of focused, radially polarized fields is analyzed. It is shown that the focal region contains different kinds of polarization singularities such as $L$-lines, stars, monstars, lemons, and $V$-points. Their interrelation is examined, and it is demonstrated how polarization singularities can be created or annihilated when, e.g., the semi-aperture angle of the focusing system is changed.

\section{Focused, radially polarized fields}

Consider an aplanatic focusing system $L$, as depicted in Fig. 1. The system has a focal length $f$ and a semi-aperture angle $\alpha$. The origin $O$ of a right-handed cartesian coordinate system is taken to be at the geometrical focus. A monochromatic, radially polarized beam is incident on the system. The electric and magnetic fields at time $t$ at position $\mathbf{r}$ are given by the expressions

$\# 70204$ - \$15.00 USD

(C) 2006 OSA
Received 24 April 2006; revised 17 May 2006; accepted 17 May 2006 12 June 2006 / Vol. 14, No. 12 / OPTICS EXPRESS 5734 


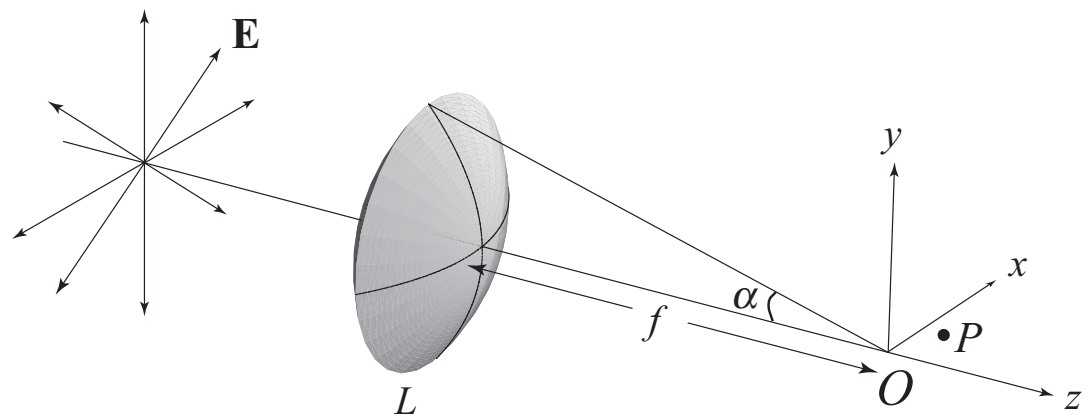

Fig. 1. Illustration of a high numerical aperture system focusing a radially polarized beam.

$$
\begin{aligned}
\mathbf{E}(\mathbf{r}, t) & =\operatorname{Re}[\mathbf{e}(\mathbf{r}) \exp (-\mathrm{i} \omega t)], \\
\mathbf{H}(\mathbf{r}, t) & =\operatorname{Re}[\mathbf{h}(\mathbf{r}) \exp (-\mathrm{i} \omega t)],
\end{aligned}
$$

respectively, where Re denotes the real part. The longitudinal component $e_{z}$ and the radial component $e_{\rho}$ of the electric field at a point $P=\left(\rho_{P}, z_{P}\right)$ in the focal region are given by the equations [19]

$$
\begin{aligned}
e_{z}\left(\rho_{P}, z_{P}\right)= & -\mathrm{i} k f \int_{0}^{\alpha} l(\theta) \sin ^{2} \theta \cos ^{1 / 2} \theta \\
& \times \exp \left(\mathrm{i} k z_{P} \cos \theta\right) J_{0}\left(k \rho_{P} \sin \theta\right) \mathrm{d} \theta, \\
e_{\rho}\left(\rho_{P}, z_{P}\right)= & -k f \int_{0}^{\alpha} l(\theta) \sin \theta \cos ^{3 / 2} \theta \\
& \times \exp \left(\mathrm{i} k z_{P} \cos \theta\right) J_{1}\left(k \rho_{P} \sin \theta\right) \mathrm{d} \theta,
\end{aligned}
$$

where $J_{i}$ is the Bessel function of the first kind of order $i$. Also, $l(\theta)$ denotes the angular amplitude function

$$
l(\theta)=f \sin \theta \exp \left[-f^{2} \sin ^{2} \theta / w_{0}^{2}\right]
$$

where $w_{0}$ is the spot size of the beam in the waist plane, which is assumed to coincide with the entrance plane of the focusing system. As stated above, the electric field has no azimuthal component. However, the magnetic field in the focal region, which we will not investigate here, is purely azimuthal. On using the dimensionless optical coordinates (sometimes referred to as Lommel variables)

$$
\begin{aligned}
& u=k z_{P} \sin ^{2} \alpha, \\
& v=k \rho_{P} \sin \alpha,
\end{aligned}
$$

to specify the position of the observation point $P$, Eqs. (3) and (4) can be rewritten as

$$
\begin{aligned}
e_{z}(u, v)= & -\mathrm{i} k f^{2} \int_{0}^{\alpha} \sin ^{3} \theta \cos ^{1 / 2} \theta \exp \left(-\beta^{2} \sin ^{2} \theta\right) \\
& \times \exp \left(\mathrm{i} u \cos \theta / \sin ^{2} \alpha\right) J_{0}\left(\frac{v \sin \theta}{\sin \alpha}\right) \mathrm{d} \theta \\
e_{\rho}(u, v)= & -k f^{2} \int_{0}^{\alpha} \sin ^{2} \theta \cos ^{3 / 2} \theta \exp \left(-\beta^{2} \sin ^{2} \theta\right) \\
& \times \exp \left(\mathrm{i} u \cos \theta / \sin ^{2} \alpha\right) J_{1}\left(\frac{v \sin \theta}{\sin \alpha}\right) \mathrm{d} \theta,
\end{aligned}
$$

$\# 70204$ - \$15.00 USD

(C) 2006 OSA 
where the parameter $\beta=f / w_{0}$ denotes the ratio of the focal length of the system and the spot size of the beam in the waist plane.

\section{The state of polarization}

The standard description of the state of polarization in terms of Stokes parameters [13] applies to plane waves, i.e. to fields in which the electric field only has two non-zero cartesian components, both perpendicular to the direction of propagation. On focusing a plane wave, the electric field acquires a third non-zero component which is directed along the direction of propagation (the so-called longitudinal field component) [21]. Under the assumption of paraxiality (i.e., assuming the semi-aperture angle of the focusing system to be small), this third component may be neglected. The configuration that we examine is not a paraxial one, but in cylindrical coordinates only two components of the electric field are non-zero. This means that with a suitable change in the definition of the Stokes parameters, the usual description of the state of polarization and, in particular, of polarization singularities can be applied.

The electric field in the focal region is given by the formula

$$
\mathbf{e}(u, v)=e_{z}(u, v) \hat{\mathbf{z}}+e_{\rho}(u, v) \hat{\boldsymbol{\rho}},
$$

with the components $e_{z}(u, v)$ and $e_{\rho}(u, v)$ given by Eqs. (8) and (9), and with $\hat{\mathbf{z}}$ and $\hat{\boldsymbol{\rho}}$ unit vectors in the longitudinal and radial direction, respectively. Let us define the variables

$$
\begin{aligned}
a_{1} & =\left|e_{z}(u, v)\right|, \\
\delta_{1} & =\arg \left[e_{z}(u, v)\right], \\
a_{2} & =\left|e_{\rho}(u, v)\right|, \\
\delta_{2} & =\arg \left[e_{\rho}(u, v)\right] .
\end{aligned}
$$

The state of polarization of the field may then be characterized by the four Stokes parameters (cf. [13, Sec. 1.4] for a similar definition with respect to a cartesian coordinate system.)

$$
\begin{aligned}
& S_{0}=a_{1}^{2}+a_{2}^{2}, \\
& S_{1}=a_{1}^{2}-a_{2}^{2}, \\
& S_{2}=2 a_{1} a_{2} \cos \delta, \\
& S_{3}=2 a_{1} a_{2} \sin \delta,
\end{aligned}
$$

with the phase difference $\delta$ given by

$$
\delta=\delta_{2}-\delta_{1}
$$

For any given intensity (i.e., $S_{0}=$ constant), the normalized Stokes parameters $s_{1}=S_{1} / S_{0}$, $s_{2}=S_{2} / S_{0}$ and $s_{3}=S_{3} / S_{0}$ may be represented as a point on the Poincaré sphere (see Fig. 2). On the north pole $\left(s_{1}=s_{2}=0, s_{3}=1\right)$, the polarization is circular. We adopt the convention of calling this state right-handed because, according to Eq. (1), the ellipse is being traversed in a clockwise manner in the $\left(e_{z}, e_{\rho}\right)$-plane. The polarization is right-handed for points on the northern hemisphere, and left-handed on the southern hemisphere. It easily verified that at the south pole $\left(s_{1}=s_{2}=0, s_{3}=-1,\right)$ the polarization is circular and left-handed. For all points on the equator $\left(s_{3}=0\right)$, the polarization is linear. At $\left(s_{1}=1, s_{2}=s_{3}=0\right)$ the field is purely $z$ polarized, whereas at $\left(s_{1}=-1, s_{2}=s_{3}=0\right)$ it is purely $\rho$-polarized. At $\left(s_{1}=0, s_{2}=1, s_{3}=0\right)$ and $\left(s_{1}=0, s_{2}=-1, s_{3}=0\right)$, finally, the linear polarization is under angle of $+\pi / 4$ and $-\pi / 4$ in the $\left(e_{z}, e_{\rho}\right)$-plane, respectively. For the case that $a_{1} \geq a_{2}$, the orientation of the ellipse is

$\# 70204$ - \$15.00 USD

(C) 2006 OSA
Received 24 April 2006; revised 17 May 2006; accepted 17 May 2006 12 June 2006 / Vol. 14, No. 12 / OPTICS EXPRESS 5736 
described by the angle $\psi$ between the major semiaxis and the $z$-direction. It is given by the expression [13, Sec. 1.4.2, Eq. 46]

$$
\psi=\frac{1}{2} \arctan \left(\frac{s_{2}}{s_{1}}\right) .
$$

It follows from Eqs. (8) and (9) that at any two points $(u, v)$ and $(-u, v)$ that are symmetrically located with respect to the focal plane, the field components satisfy the symmetry relations

$$
\begin{aligned}
e_{z}(-u, v) & =-e_{z}^{*}(u, v), \\
e_{\rho}(-u, v) & =e_{\rho}^{*}(u, v),
\end{aligned}
$$

where the asterisk denotes complex conjugation. Clearly, the variables $a_{1}$ and $a_{2}$ remain unchanged under reflection of the point of observation in the focal plane. The behavior of the other quantities that describe the state of polarization is summarized in Table 1. We note that the antisymmetrical behavior of the second Stokes parameter and the angle $\psi$ implies that they both vanishes identically in the focal plane, i.e.,

$$
s_{2}(0, v)=0 ; \quad \psi(0, v)=0 .
$$

The symmetry properties of the polarization ellipse are illustrated in Fig. 3. If, for example, the major semiaxis of the polarization ellipse at a point $(z, x)$ makes an angle $\psi$ with the positive $z$-axis, then at a point $(-z, x)$ the orientation angle will be $-\psi$. The handedness, however, is the same at both positions. The orientation of the ellipse and its handedness at $(z,-x)$ and $(-z,-x)$ follow from considering the rotational symmetry of the field.

An example of the evolution of the Stokes parameters $s_{1}$ and $s_{3}$ in the focal plane as the radial distance $v$ is increased is shown in Fig./Movie 4. Using the relation $s_{1}^{2}+s_{2}^{2}+s_{3}^{2}=1$ together

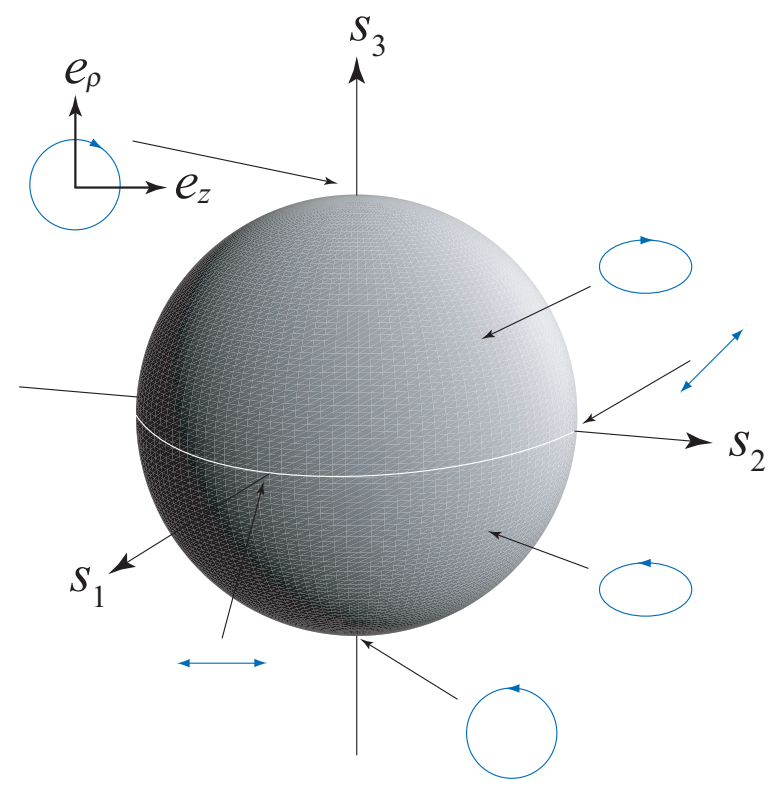

Fig. 2. The Poincaré sphere with cartesian axes $\left(s_{1}, s_{2}, s_{3}\right)$.

\#70204 - \$15.00 USD

(C) 2006 OSA
Received 24 April 2006; revised 17 May 2006; accepted 17 May 2006 12 June 2006 / Vol. 14, No. 12 / OPTICS EXPRESS 5737 


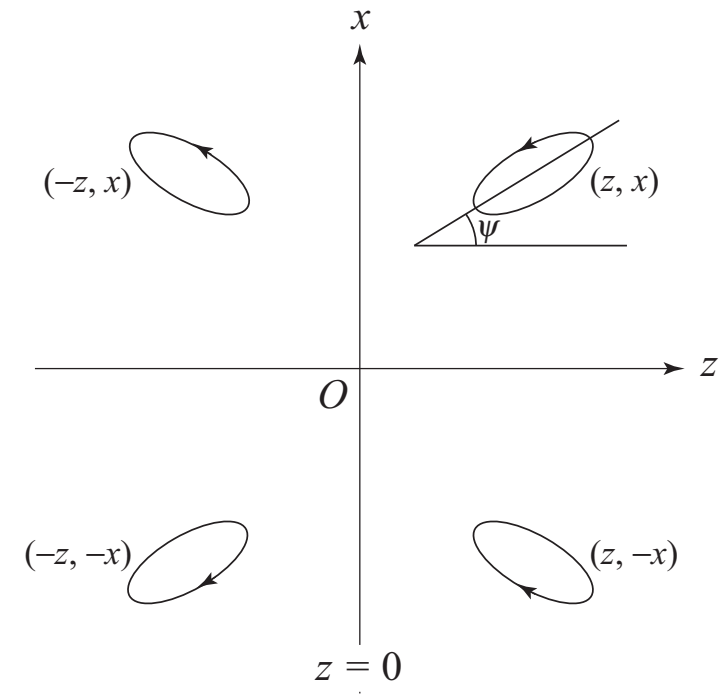

Fig. 3. Illustrating the symmetry properties of the polarization ellipse.

with the first expression in (23), it is found that for observation points in the focal plane the $\left(s_{1}, s_{3}\right)$ vector has unit length. When the radial distance is increased, the state of polarization is seen to exhibit a cyclical behavior, changing from linear to circular and back to linear again. In other words, the Stokes vector traverses the $s_{2}=0$ meridian of the Poincaré sphere several times.

\section{Polarization singularities}

At points where the polarization ellipse degenerates into a circle or into a line, the polarization is said to be singular. At $C$-points (i.e., $s_{3}= \pm 1$ ), where the polarization is circular, the orientation angle $\psi$ of the ellipse, as given by Eq. (20), is undetermined. At $C$-points the polarization can either be left-handed or right-handed. At $L$-lines (i.e., $s_{3}=0$ ), where the polarization is linear, the handedness of the ellipse is undetermined. In the remainder these two types of polarization singularities are examined. It should be noted that, because of rotational symmetry, points and closed lines in the $(u, v)$-plane are circles and tori, respectively, in three-dimensional space. We remark in passing that it should be clear from the above that at polarization singularities the state of polarization is well-defined.

Table 1. The behavior of various quantities that characterize the state of polarization under reflection of the point of observation in the focal plane.

\begin{tabular}{|c||c|c|c|c|c|c|c|c|}
\hline$(\mathrm{u}, \mathrm{v})$ & $\delta_{1}$ & $\delta_{2}$ & $\delta$ & $s_{0}$ & $s_{1}$ & $s_{2}$ & $s_{3}$ & $\psi$ \\
\hline \hline$(-\mathrm{u}, \mathrm{v})$ & $\pi-\delta_{1}$ & $-\delta_{2}$ & $-\delta-\pi$ & $s_{0}$ & $s_{1}$ & $-s_{2}$ & $s_{3}$ & $-\psi$ \\
\hline
\end{tabular}

\#70204 - \$15.00 USD

(C) 2006 OSA
Received 24 April 2006; revised 17 May 2006; accepted 17 May 2006 12 June 2006 / Vol. 14, No. 12 / OPTICS EXPRESS 5738 


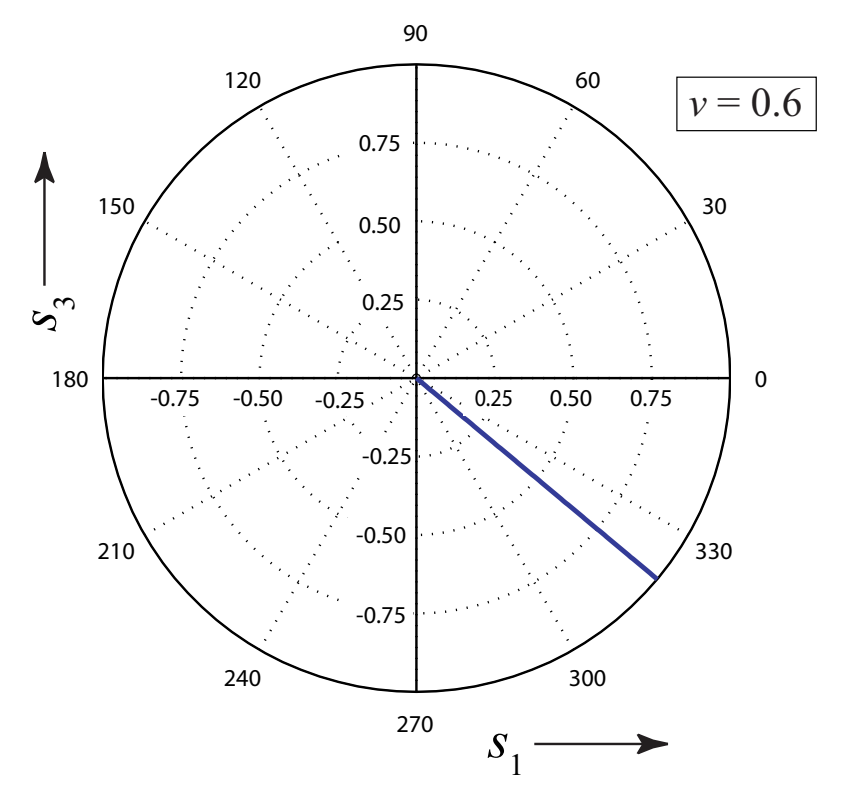

Fig. 4. The normalized Stokes parameters $s_{1}$ and $s_{3}$ in the focal plane for increasing values of the dimensionless radial distance $v$. In this example $\alpha=\pi / 4$ and $\beta=1.5$.

\subsection{Linear polarization}

It follows from Eq. (18) that linear polarization generically occurs at points $(u, v)$ at which

$$
\operatorname{Re}\left[e_{z}(u, v)\right] \operatorname{Im}\left[e_{\rho}(u, v)\right]=\operatorname{Im}\left[e_{z}(u, v)\right] \operatorname{Re}\left[e_{\rho}(u, v)\right] .
$$

This single condition is typically satisfied on a line in $(u, v)$-space. A subset of these points are locations where one of the two field components is zero, i.e. at phase singularities of either field component. For example, for the longitudinal component these occur at points at which

$$
\operatorname{Re}\left[e_{z}(u, v)\right]=\operatorname{Im}\left[e_{z}(u, v)\right]=0 .
$$

These two conditions are typically satisfied at isolated points in $(u, v)$-space. Phase singularities of both field components are found in the focal plane and at other points in space. They can be created or annihilated by smoothly varying the width of the incident beam or the semi-aperture angle [20].

By drawing the contours of $s_{3}=0$, a multitude of $L$-surfaces is found. As can be seen in Fig. 5, the phase singularities of the two electric field components all lie on $L$-surfaces. Notice that there is a surface of linear polarization that connects each Airy ring of $e_{z}$ in the focal plane to the adjacent Airy ring of $e_{\rho}$. On traversing these closed surfaces in the $u, v$-plane, the Stokes vector makes a complete rotatation along the equator of the Poincare sphere.

It is seen from Eq. (9) that on the central axis (i.e., $v=0$ ) the radial electric field component vanishes. Therefore this axis constitutes an $L$-line. It follows from rotational symmetry that its index is +1 (see [2, Sec. 13.3]). 


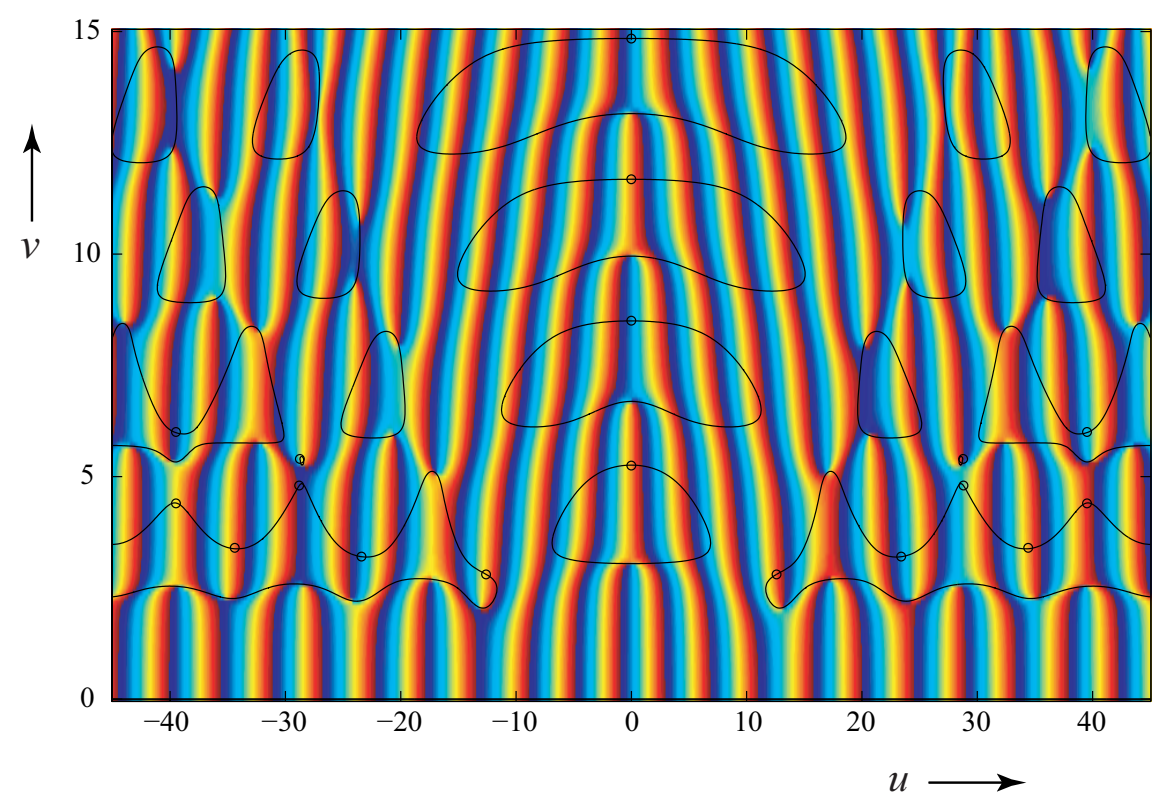

Fig. 5. The loci of linear polarization, i.e. contours of $s_{3}=0$, in the focal region. Because of the rotational symmetry of the field, these are tori centered on the $u$-axis. The contours are superposed on a color-coded phase map of $e_{z}$. Phase singularities of $e_{z}$ are located at points where all different colors converge. The open circles indicate phase singularities of the other field component, $e_{\rho}$. In this example $\alpha=\pi / 4$ and $\beta=0.5$.

\subsection{Circular polarization}

It follows from the definitions (16)-(18) that circular polarization generically occurs at points rather than lines in the $u, v$-plane. One way of locating $C$-points is to represent the field in a circular polarization basis (cf. [22] for an similar decomposition in cartesian coordinates), i.e.,

$$
\mathbf{e}(u, v)=e_{+}(u, v) \hat{\mathbf{c}}_{+}(u, v)+e_{-}(u, v) \hat{\mathbf{c}}_{-}(u, v),
$$

where

$$
e_{ \pm}=\left(e_{z} \mp \mathrm{i} e_{\rho}\right) / \sqrt{2}, \quad \hat{\mathbf{c}}_{ \pm}=(\hat{z} \pm \mathrm{i} \hat{\rho}) / \sqrt{2} .
$$

Thus $e_{+}\left(e_{-}\right)$represents the amplitude of the right- (left-) handed circular component of the field. In this way, $C$-points correspond to phase singularities of either circular component. An example is presented in Fig. 6. It is seen that the number of right- and left-handed $C$-points is approximately the same. However, there is a line (a cylindrical sheet in three-dimensional space) of left-handed circular polarization at approximately $v=1.4$. This is a non-generic surface that appears to be only weakly dependent on the parameters $u$ and $\beta$. To understand this feature more fully we apply a first-order Taylor expansion in $\theta$ to Eqs. (8) and (9). These expressions then become

$$
\begin{aligned}
& e_{z}(u, v)=-\mathrm{i} k f^{2} \int_{0}^{\alpha} \theta^{3}\left(1-\beta^{2} \theta^{2}\right) \exp \left(\mathrm{i} u / \sin ^{2} \alpha\right) \mathrm{d} \theta \\
& e_{\rho}(u, v)=-k f^{2} \int_{0}^{\alpha} \theta^{3}\left(1-\beta^{2} \theta^{2}\right) \exp \left(\mathrm{i} u / \sin ^{2} \alpha\right) \frac{v}{2 \sin \alpha} \mathrm{d} \theta .
\end{aligned}
$$

\#70204 - \$15.00 USD

(C) 2006 OSA 
Thus, $e_{+}$can be written as

$$
e_{+}(u, v)=-\frac{\mathrm{i} k f^{2}}{\sqrt{2}} \exp \left(\mathrm{i} u / \sin ^{2} \alpha\right)\left[1-\frac{v}{2 \sin \alpha}\right] \int_{0}^{\alpha} \theta^{3}\left(1-\beta^{2} \theta^{2}\right) \mathrm{d} \theta .
$$

It is readily apparent that left-handed circular polarization occurs when $v=2 \sin \alpha$. Also, because the zero of $e_{+}$is independent of the longitudinal variable $u$, this phase singularity is lineshaped. Notice that there is no corresponding $C$-surface for right-handed circular polarization because the resulting equation would yield (at first order) $v=-2 \sin \alpha$, whereas $v$ is positive definite. To test the validity of this approximation, the computed location of the $C$-surface is compared to the approximate values in Table 2. Even when the semi-aperture angle $\alpha=60^{\circ}$, the approximation is seen to hold quite well.

(a)

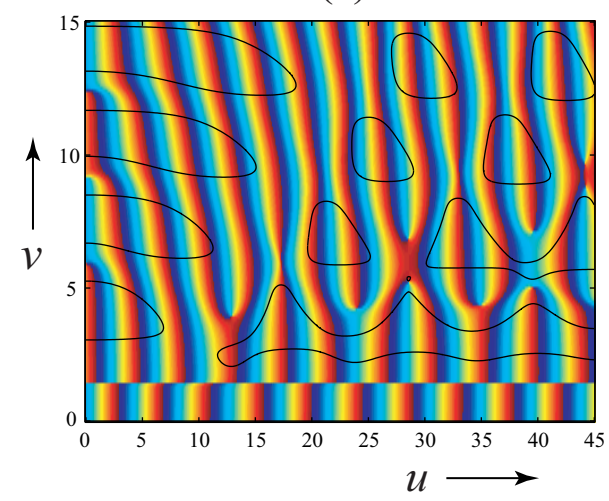

(b)

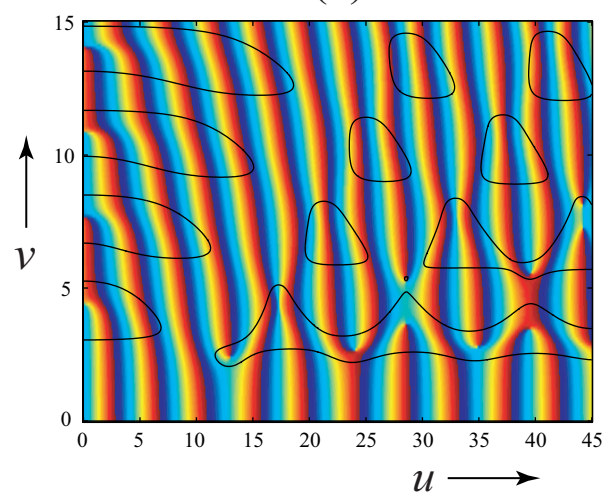

Fig. 6. A color-coded phase map of (a) $e_{+}$and (b) $e_{-}$, both with lines of linear polarization (solid black curves) superposed. Left-handed $C$-points are phase singularities of $e_{+}$in panel (a), whereas right-handed $C$-points are singularities of $e_{-}$in panel (b). In this example $\beta=0.5$ and $\alpha=\pi / 4$.

Apart from their handedness, $C$-points can be classified into three distinct types based on the local behavior of the polarization ellipses (see [2, Secs. 4.10 and 12.2] and Ref. [23]). These types are stars (with index $-1 / 2$ ), monstars (index 1/2), and lemons (index 1/2). This local behavior is shown in Fig. 7 for the pair of $C$-points seen in Fig. $6 \mathrm{~b}$ near $(u, v) \approx(28,5)$ for three different values of the semi-aperture angle $\alpha$. Indicated in red are the local straight-line orientations of the major axis of the nearby ellipses. (A single line for lemons, and three lines

Table 2. Comparison of the approximate location of the $C$-surface to its actual location. In these examples $\beta=0.5$.

\begin{tabular}{|c|c|c|c|}
\hline$\alpha$ & actual $v$ & $v=2 \sin \alpha$ & Error \\
\hline \hline $15^{\circ}$ & 0.515 & 0.517 & $0.50 \%$ \\
\hline \hline $30^{\circ}$ & 1.005 & 1.000 & $0.50 \%$ \\
\hline \hline $45^{\circ}$ & 1.425 & 1.414 & $0.76 \%$ \\
\hline \hline $60^{\circ}$ & 1.765 & 1.732 & $1.87 \%$ \\
\hline
\end{tabular}

\#70204 - \$15.00 USD

(C) 2006 OSA
Received 24 April 2006; revised 17 May 2006; accepted 17 May 2006 12 June 2006 / Vol. 14, No. 12 / OPTICS EXPRESS 5741 
(a)

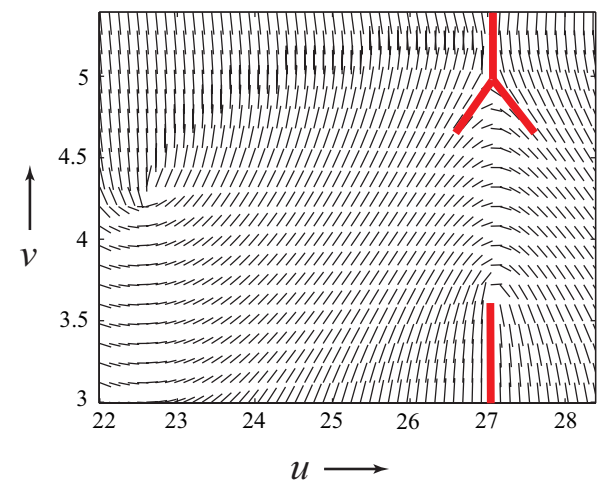

(b)

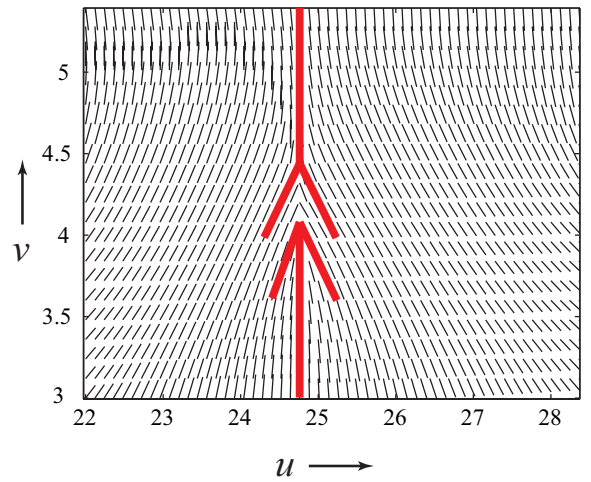

(c)

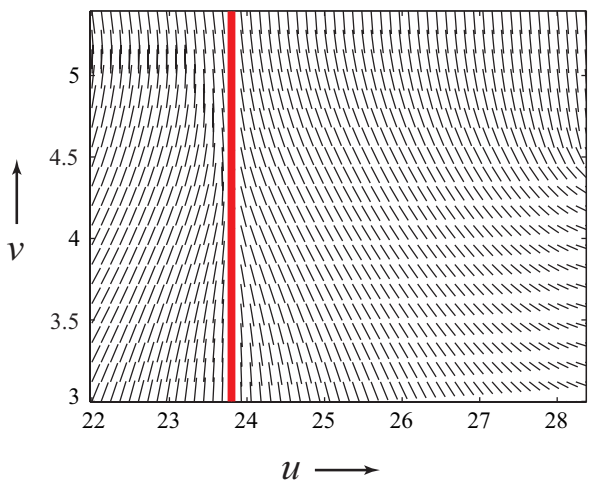

Fig. 7. Local orientation of the major axis of the electric polarization ellipse shown for three different values of the semi-aperture angle $\alpha$ with the beam parameter $\beta$ kept fixed at 0.5 . The local straight-line orientations of the major axes are shown in red to aid the eye. In panel a $\left(\alpha=52^{\circ}\right)$, a star (above) and a lemon (below) are seen. In panel b $\left(\alpha=61^{\circ}\right)$, the lemon has transitioned into a monstar. In panel c $\left(\alpha=65^{\circ}\right)$, the annihilation leaves only a straight-line orientation of the major axis.

for stars and monstars). In Fig. 7(a) both a star (top) and a lemon (bottom) can be seen. The lemon-type polarization singularity has evolved into a monstar in Fig. 7(b). In panel 7(c) the situation is shown after the star and the monstar have annihilated.

\subsection{Relationship between L-lines and C-points}

There is a strong connection between $L$-lines and $C$-points. The former separate space into regions of different handedness. In agreement with this, the left-handed $C$-points in Fig. 6(a), are all located outside the closed $L$-lines, whereas the right-handed $C$-points in Fig. 6(b) are all located within them. As reported by Freund et al. [16], there is a relation between the charge of component singularities on a closed $L$-line and the total charge of $C$-points enclosed by it. For our specific case Eq. (5) of [16] can be re-written as

$$
2 \sum_{\in L} q_{-}=\sum_{L} q_{\rho}+\sum_{L} q_{z}
$$

\#70204 - \$15.00 USD

(C) 2006 OSA
Received 24 April 2006; revised 17 May 2006; accepted 17 May 2006 12 June 2006 / Vol. 14, No. 12 / OPTICS EXPRESS 5742 
where the summation on the left-hand side is over all topological charges of $C$-points enclosed by the $L$-line, and the right-hand summations are over the charges of the two electric field components on the $L$-line. For example, it was verified that the two $L$-lines that contain the points $(0,4)$ and $(22,7)$ in Figs. 5 and 6 both satisfy this sum rule.

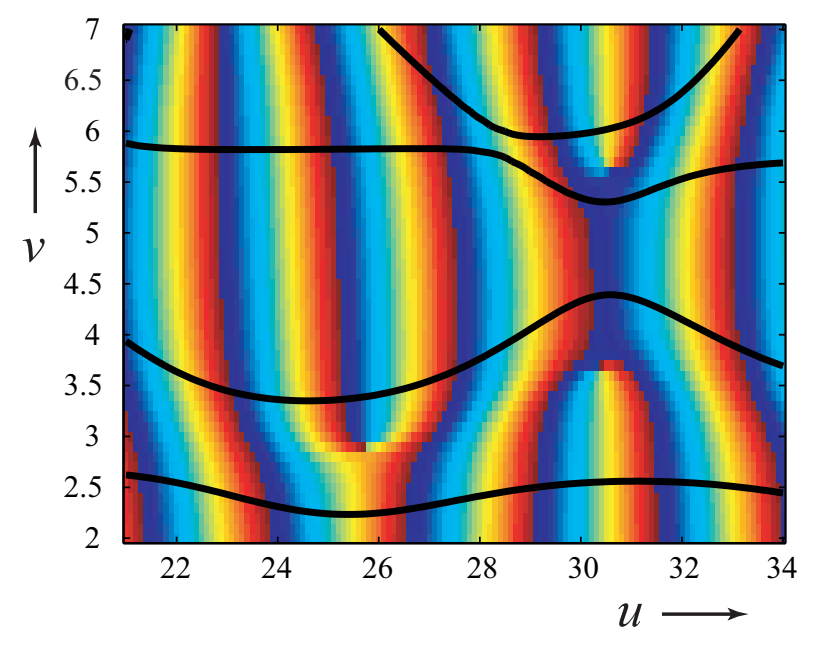

Fig. 8. A color-coded phase map of the left-handed component $e_{-}$with $L$-lines, shown in black, superposed. The semi-aperture angle $\alpha$ is increased from $35^{\circ}$ to $65.5^{\circ}$, with $\beta$ kept fixed at 0.5 .

Figure/Movie 8 shows the evolution of the left-handed field component $e_{-}$and the $L$-lines as the semi-aperture angle $\alpha$ is increased. Notice that $L$-lines deform, separate, and merge. A small $L$-line that contains a $C$-point in its interior can be seen to break off when $\alpha \approx 43^{\circ}$, and unite with another $L$-line when $\alpha \approx 46.5^{\circ}$. In addition, a $C$-point annihilation is seen near $\alpha \approx 61^{\circ}$. The upper singularity is a star and the lower singularity is a lemon that evolves into a monstar before the annihilation takes place. Fig. 7 shows the corresponding local ellipse behavior for this event.

\subsection{Vector singularities}

In addition to the different kinds of singularities mentioned in Sec. 1, there exist also points where the two-dimensional complex electric vector field $\mathbf{e}$ is identically zero. Such points are referred to as $V$-points [24]. $V$-points are necessarily a phase singularity of both cylindrical components and of both components in the circular polarization basis. They are to be distinguished from singularities of real-valued vector fields such as the Poynting vector [5]-[7]. Since the complex electric field given by Eqs. (8) and (9) is an analytic function, its zeros are isolated points in the $(u, v)$ plane [25]. Because the condition $\mathbf{e}(u, v)=0$ has co-dimension four, $V$ points do not generically occur. However, as we now demonstrate, they do appear in the focal region of radially polarized beams.

In our configuration $V$-points occur, for example, when the longitudinal component $e_{z}$ is zero on the optical axis (where the radial component $e_{\rho}$ is identically zero). In Fig./Movie 9 the real and imaginary parts of $e_{z}$ are shown. Their intersection near $u=11.5$ is seen to move towards and eventually below the horizontal axis when the semi-aperture angle $\alpha$ is increased in a continuous manner. At approximately $\alpha=56.6^{\circ}$, this intersection crosses the horizontal 
axis, i.e. $\operatorname{Re}\left(e_{z}\right)=\operatorname{Im}\left(e_{z}\right)=0$ near $(u, v)=(11.5,0)$. Clearly this $V$-point is unstable under perturbations.

Another way in which vector singularities may occur is through the collision of an $L$-line and a $C$-point [14]. Although the field at a $V$-point is neither linearly nor circularly polarized, the condition for linear polarization, $\left(S_{3}=0\right)$ combined with the two conditions for circular polarization, $\left(S_{1}=S_{2}=0\right)$, result in the necessary condition that $S_{0}=0$, i.e. the electric field vanishes at the collision point. An example of such an event is shown in Fig./Movie 10 in which the semi-aperture angle $\alpha$ is gradually increased. The $L$-line that is seen to break apart moves downwards towards the horizontal $C$-line near $v=1$.6. In this process the $L$-line shrinks and collapses to a point at the moment of collision. On further increasing $\alpha$, this point changes back into an $L$-line that approaches the optical axis. The $C$-line remains essentially stationary while the $L$-line passes through it. We mention in passing that the $L$-line of interest in Fig./Movie 10 intersects one phase singularity of each cylindrical component and encloses one point of pure right-handed circular polarization. When the $L$-line collapses to a point at the line of pure lefthanded polarization (at approximately $v=1.6$ ), both cylindrical singularities coincide, creating a $V$-point. Alternatively, the $V$-point can be be considered as the collision of phase singularities of the circular components $e_{+}$and $e_{-}$. In fact, because $L$-surfaces separate space into regions of right- and left-handedness, the only way in which $C$-points of opposite handedness can collide is when the $L$-line separating them collapses to a point.

\section{Discussion}

It is to be noted that in the system at hand the magnetic field in the focal region has only a single (azimuthal) component. This implies that the behavior of the magnetic polarization is trivial compared to that of the electric polarization.

Also, because of the rotational symmetry of the configuration, the closed $L$-lines that are seen in the $u, v$-plane, form tori in three-dimensional space centered on the optical axis. Likewise, the $C$-points form rings.

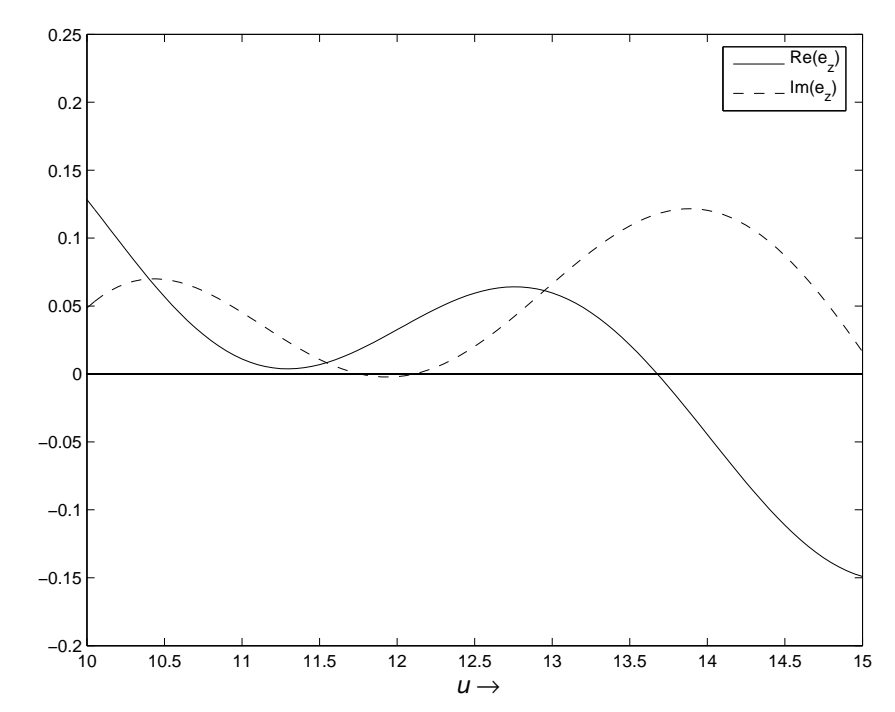

Fig. 9. The real and imaginary parts of $e_{z}$ along the optical axis normalized to $\operatorname{Im}\left[e_{z}(0,0)\right]$ as $\alpha$ ranges from $55.0^{\circ}$ to $58.0^{\circ}$, with $\beta$ kept fixed at 1.5 .

$\# 70204$ - \$15.00 USD

(C) 2006 OSA
Received 24 April 2006; revised 17 May 2006; accepted 17 May 2006 12 June 2006 / Vol. 14, No. 12 / OPTICS EXPRESS 5744 


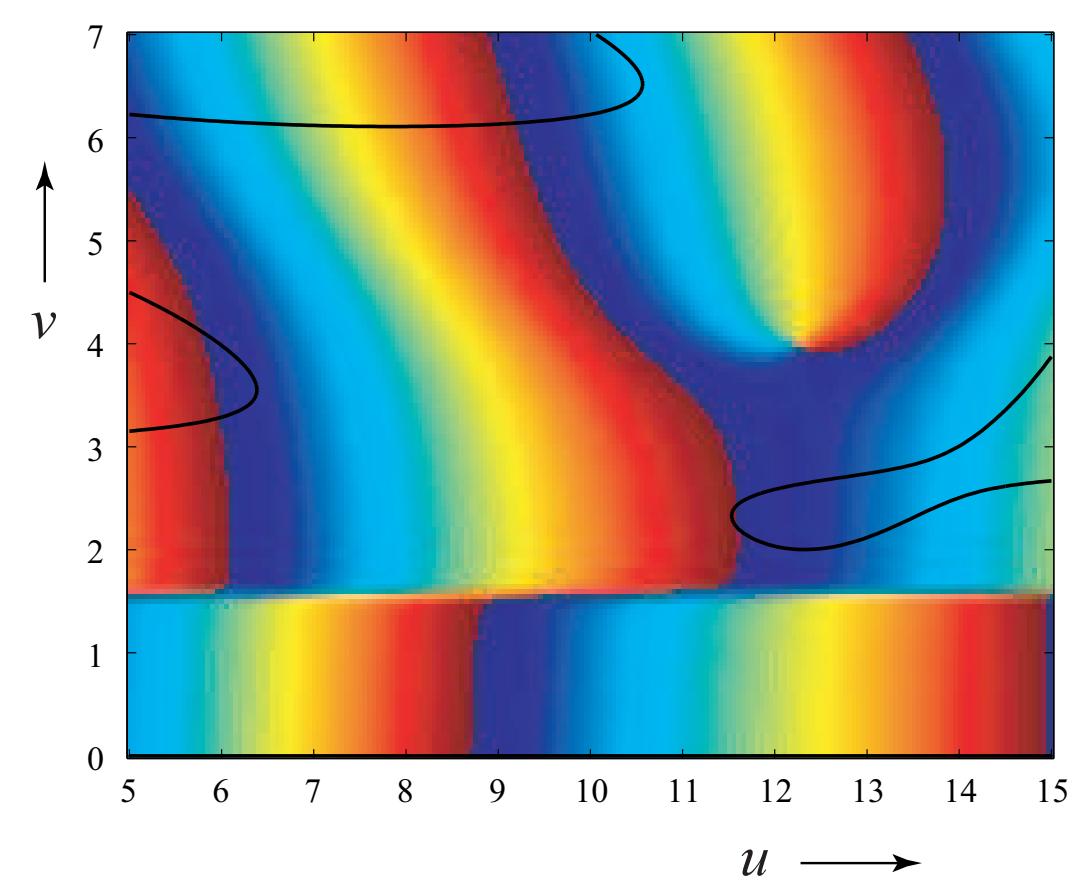

Fig. 10. A color-coded phase map of $e_{+}$with $L$-lines (solid black curves) superposed as the semi-aperture angle $\alpha$ ranges from $50.0^{\circ}$ to $66.0^{\circ}$ and with $\beta$ kept fixed at 1.5.

In summary, strongly focused, radially polarized fields have been considered within the context of singular optics. The state of polarization was discussed and analyzed for this system. Phase singularities of the two electric field components and polarization singularities were identified. The relation between $L$-lines and $C$-lines was discussed, and an annihilation of two $C$-points involving a star, a lemon and a monstar, was shown. Finally, it was demonstrated that isolated vector singularities, $V$-points, can occur in this system and two different ways of creating them were analyzed.

\section{Acknowledgments}

The first author acknowledges the Fulbright Center for their financial support, the second author wishes to thank Prof. Emil Wolf for stimulating discussions.

$\# 70204$ - \$15.00 USD

(C) 2006 OSA
Received 24 April 2006; revised 17 May 2006; accepted 17 May 2006

12 June 2006 / Vol. 14, No. 12 / OPTICS EXPRESS 5745 